\title{
Highly Polarized Multiwavelength Er-doped Fibre Laser Using All Fibre Lyot Filter
}

\author{
Zihao Zhao ${ }^{(1)}$, Yue Li (1), Zhijun Yan ${ }^{(1),(2),(3)}$, Yiyang Luo ${ }^{(1)}$, Hushan Wang(3), Qizhen Sun ${ }^{(1),(2)}$, \\ Deming Liu ${ }^{(1)}$, and Lin Zhang ${ }^{(2)}$
}

(1) The School of Optical and Electronic Information, National Engineering Laboratory for Next Generation Internet Access System, Huazhong University of Science and Technology, Wuhan 430074, Hubei, P. R. China, yanzhijun@hust.edu.cn

(2) Aston Institute of Photonic Technologies, Aston University, Birmingham, UK, B4 7ET

(3) State Key Laboratory of Transient Optics and Photonics, Xi'an Institute of Optics and Precision Mechanics, Chinese Academy of Sciences, Xi'an, China, 710119

\begin{abstract}
A multiwavelength Er-doped fibre ring laser system using all fibre Lyot filter and highly nonlinear fibre was experimentally demonstrated, with degree of polarization up to $99.9 \%$ and 50 channels output.
\end{abstract}

Keywords: fiber laser, Polarization, Lyot filter

\section{INTRODUCTION}

Multiwavelength fibre laser has been of great interested for its widespread applications in optical communication, fibre optical sensing and optical instrumentation. In the past decades, varies of filtering techniques were employed such as Fabry-Perot Filters (FPFs)[1], cascading Fibre Bragg Gratings (FBGs) and Sagnac loop mirrors. However, these solutions have their limitations for either having random polarization output or requiring sophisticated setup. In this paper, we employed an all fibre Lyot filter as a comb filter for wavelength selection. Besides, due to the principle of Lyot filter, it also functionalized as a polarization selector, resulting highly single polarized multiwavelength output. 50 wavelengths lasing simultaneously with wavelength space of $\sim 0.162 \mathrm{~nm}$ was obtained. Degree of polarization (DOP) of the lasing channels reached up to $99.9 \%$.

\section{EXPERIMENTAL SETUP AND PRINCIPLE}

All fibre Lyot filter was implemented by alternatively concatenating $45^{\circ}$ tilted fibre gratings (TFGs) and polarization maintaining (PM) fibre cavities with axis $45^{\circ}$ to each other. The FSR and bandwidth of this Lyot filter can be expressed as:

$$
F S R \cong \frac{\lambda^{2}}{L \Delta n}, \quad \text { bandwidth }=\frac{\lambda^{2}}{2 L \Delta n}
$$

Where, L ( $46 \mathrm{~m})$ was the length of the PM fibre cavities of the filter, $\Delta \mathrm{n}\left(3.27 \times 10^{-4}\right)$ is the birefringence of $\mathrm{PM}$ fibre and $\lambda(1550 \mathrm{~nm})$ is the working wavelength. $45^{\circ}$ TFGs in the all fibre Lyot filter function as an in-finer linear polarizers[2], thus the Lyot filter has the characteristics of single polarization selection. Fig. 1 showed the transmission spectrum of the.

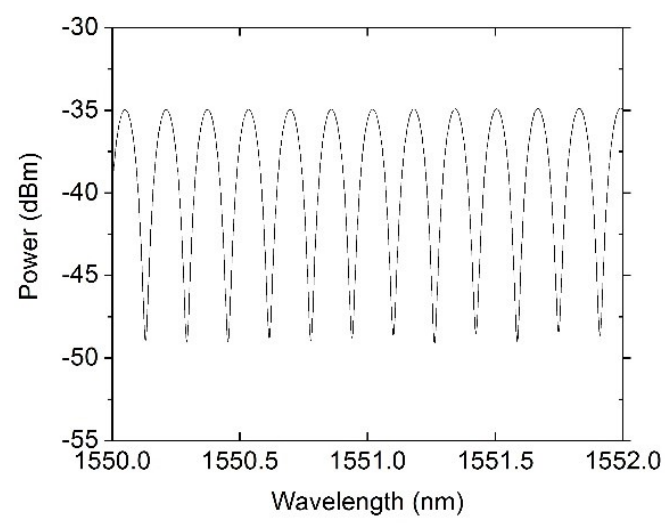

Fig. 1: Transmission spectrum of the Lyot filter.

Lyot filter, which was obtained by an ASE source and an optical spectrum analyser (YOKOGAWA AQ6370D, resolution $0.016 \mathrm{~nm})$. This Lyot filter has FSR of $\sim 0.162 \mathrm{~nm}$, bandwidth of $\sim 0.081 \mathrm{~nm}$ and extinction ratio of $\sim 14 \mathrm{~dB}$.

Fig. 2 showed the schematic of our experimental setup. The polarization independent isolator ensures the unidirectional operation of the ring cavity. The gain of the fibre laser was provided by a segment of $1.5 \mathrm{~m}$ erbiumdoped fibre (EDF, I25) pumped by a $980 \mathrm{~nm}$ laser diode (LD) pump. A section of $1 \mathrm{~km}$ highly nonlinear fibre (HNLF, dispersion of $-0.396 \mathrm{ps} / \mathrm{nm} / \mathrm{km}$ at $1545 \mathrm{~nm}$ and nonlinear coefficient of $11 / \mathrm{W} / \mathrm{km}$ ) was used to reduce the competition among longitudinal modes so that the power at the different channels could be balanced by comb filtering characteristics of Lyot filter, making it possible for multiwavelength lasing simultaneously[1]. 


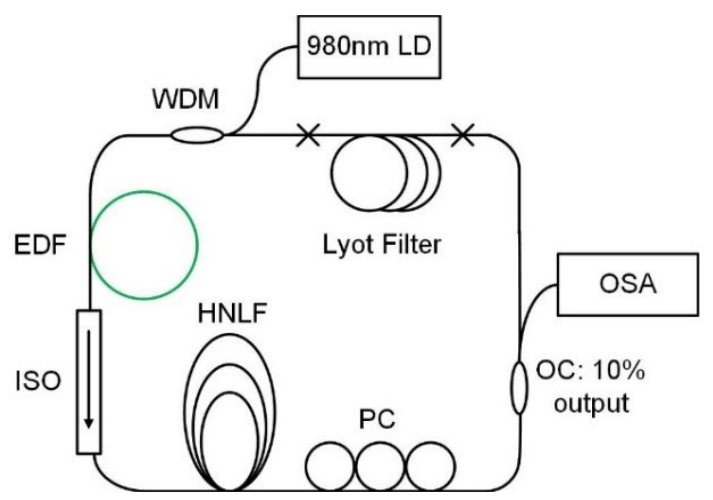

Fig. 2: Schematic of the experimental setup.

\section{RESULTS AND DISCUSSIONS}

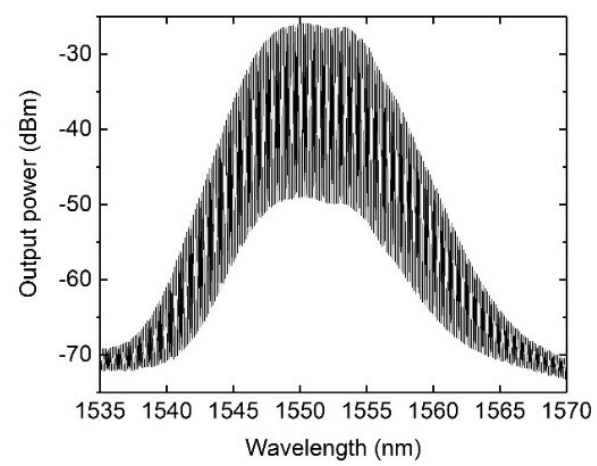

Fig. 3: 50 wavelengths lasing output.

By adjusting PC, 50 wavelengths simultaneous lasing could be easily obtained. Taking both channel quality and entirety flatness into consideration, the output had its best condition with linewidth of $0.05 \mathrm{~nm}$, side-mode suppression ratio of $20.001 \mathrm{~dB}, 50$ wavelengths lasing and $0.7341 \mathrm{~mW}$ output power when the pump power was 89.65mW, as shown in Fig. 3.
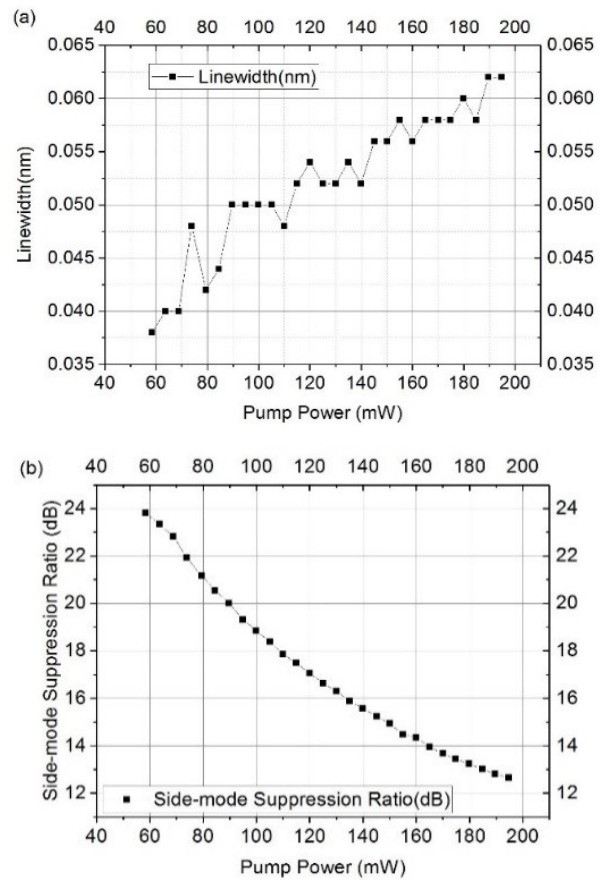

Fig. 4: Linewidth (a) and side-mode suppression ratio (b) of output laser versus pump power.

Furthermore, we have investigated the laser output characteristic with increasing the pump power. It was noticeable as increasing of the pump power, the linewidth of output laser was increasing, and the side-mode suppression ratio was decreasing (see in Fig. 4 (a) and (b)), which could be interpreted as follows: four-wave mixing (FWM) played the main role to suppress mode competition among all the nonlinear effect introduced by HNLF. Meanwhile, as pump power increases, FWM between longitudinal modes became stronger. Modes with higher power which made up the central of a channel, would share their power to their side-modes. Thus, channels got dumpy with their linewidth increasing and side-mode suppression ratio decreasing. The decreasing of linewidth at several points is because new channels started lasing[1].
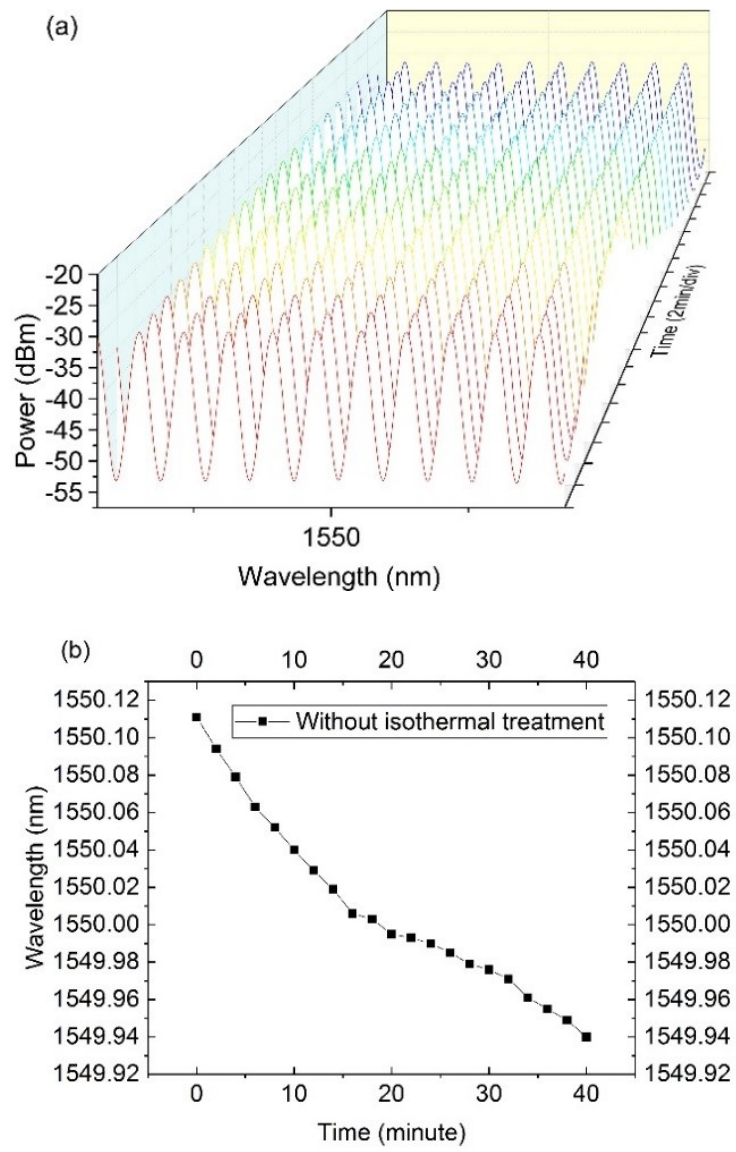

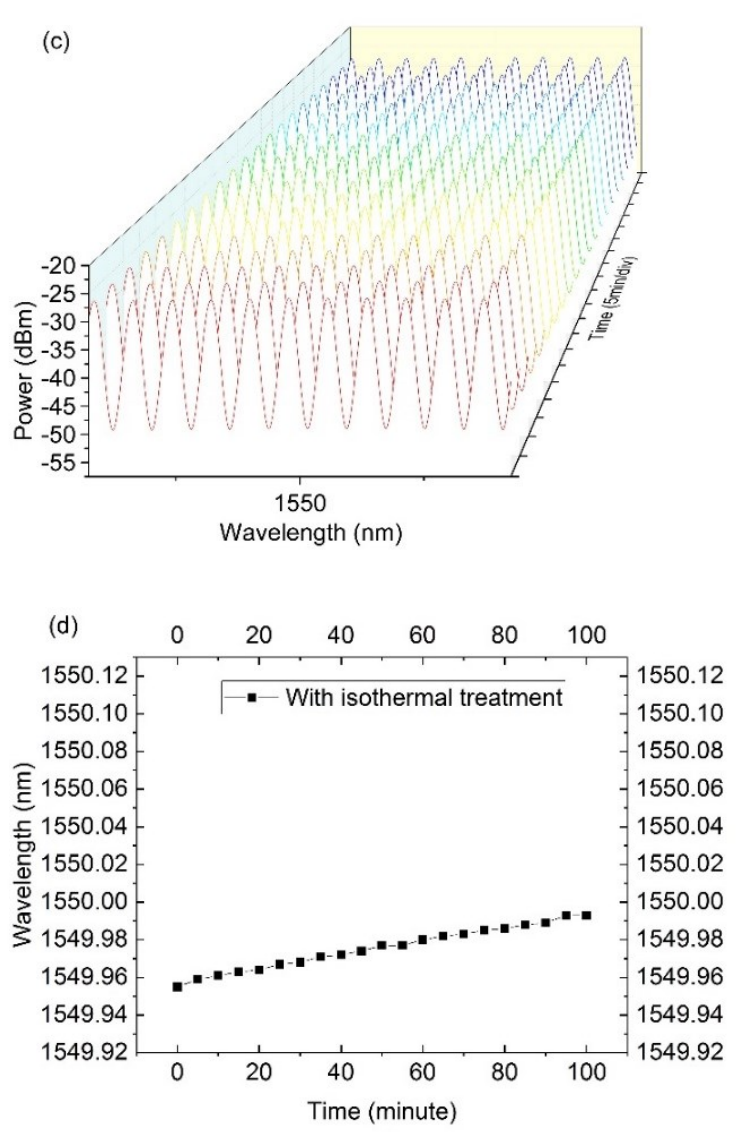

Fig. 5: Wavelength shifting without and with isothermal treatment (a), (b) and (c), (d) respectively.

The stability of output laser was measured by capturing the laser spectrum with 2-minute interval, which was shown in Fig. 5 (a). The output laser at $1550.111 \mathrm{~nm}$ shifted more than $0.2 \mathrm{~nm}$ within 40 minutes (see in Fig. 5(b)). In our previous work, we have found the Lyot filter was very sensitive to the temperature, and the longer cavity length was, the higher temperature sensitivity[3]. So, to eliminate the affect from the fluctuation of environment temperature and increase the wavelength stability of output laser, we need to keep the Lyot filter within a stable temperature environment. In the experiment, we created a thermostatic icebath to keep a constant temperature for the Lyot filter. The results showed the wavelength stability was improved greatly, which was showed in Fig. 5(c) and (d). As it shown in the figure, the output spectrum captured with 5-minute interval and the wavelength at $1549.955 \mathrm{~nm}$ shifting within 100 minutes when the Lyot filter was treated in the thermostatic icebath. The slight shift towards longer wavelength is probably because the Lyot filter did not reach thermal equilibrium yet in the isothermal system.

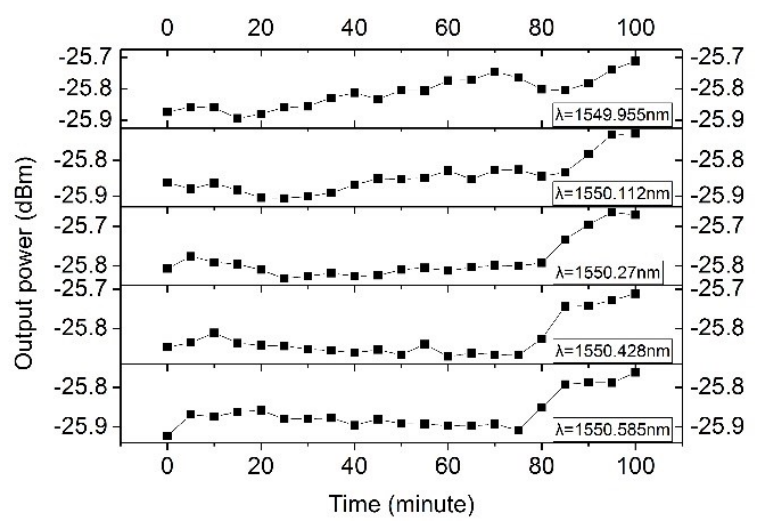

Fig. 6: Power fluctuation of five lasing channels.

Finally, we also measured the power stability and DOP of output laser. Five output laser channels were monitored for power fluctuation, which the maximum power fluctuation of each peak is less than $0.182 \mathrm{~dB}$ (see in Fig. $6)$. For the DOP measurement, we chose the output laser at $1547.25 \mathrm{~nm}, 1550.04 \mathrm{~nm}$ and $1552.75 \mathrm{~nm}$, which were $99.95 \%, 99.89 \%$ and $99.92 \%$, respectively.

\section{CONCLUSIONS}

We have experimentally demonstrated a highly polarized multiwavelength Er-doped fibre laser using all fibre Lyot filter. In the experiment, 50 wavelengths lasing simultaneously was obtained. DOP of the lasing channels reach up to $99.9 \%$. The power fluctuation of a single channel is less than $0.182 \mathrm{~dB}$. Besides, we experimentally proved that temperature was the major factor causing wavelength shifting to all fibre Lyot filter.

\section{ACKNOWLEDGEMENTS}

This work was supported by the National Natural Science Foundation of China under project (No.61505244) and (No. 61275004).

\section{REFERENCES}

[1] Shilong Pan et al., "Multiwavelength erbium-doped fibre laser based on inhomogeneous loss mechanism by use of a highly nonlinear fibre and a Fabry-Perot filter," Op. Express., Vol. 14, no. 3, February 6, 2006

[2] Zhijun Yan et al., "In-fibre linear polarizer based on UV-inscribed $45^{\circ}$ tilted grating in polarization maintaining fibre," Opt. Lett., Vol. 37, no. 18, September 15,2012

[3] Zhijun Yan et al., "Broadband Tunable All-Fibre Polarization Interference Filter Based on $45^{\circ}$ Tilted Fibre Gratings," Journal of Lightwave Technology, Vol. 31, no. 1, January 1, 2013 